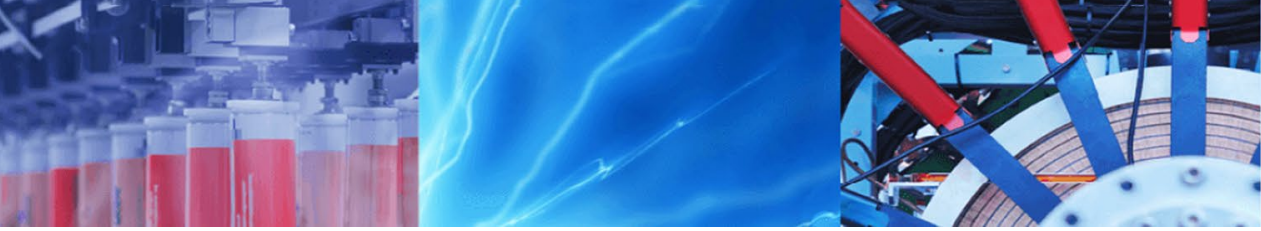

Research Article

\title{
Thermal conductivity of nanofluids formed by carbon flurooxide mesoparticles
}

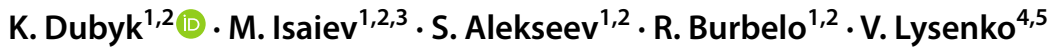

(c) Springer Nature Switzerland AG 2019

\begin{abstract}
Mesoparticles with characteristic sizes larger than all known molecules and smaller than usual nanoparticles can easily penetrate inside living cells. Thus, they appear to be especially promising for cancer theranostic application. However, presence of mesoparticles can strongly perturb physico-chemical properties of the biological media in which they are incorporated, it is crucial to know a magnitude of such perturbations. For example, thermal properties of liquid solutions with dispersed mesoparticles are crucial for development of theranostic approaches based on photoacoustic effect. In this paper, thermal transport in nanofluids formed by carbon flurooxide (CFO) mesoparticles is reported. A significant non-linear enhancement of the thermal conductivity of the nanofluids (up to $85 \%$, in comparison with initial basic liquids) depending on volume fraction of the dispersed CFO mesoparticles was found. It was revealed that the thermal conductivity rise can be explained by existence of interfacial layers between the mesoparticles and the hosting liquids.
\end{abstract}

Keywords Mesoparticles $\cdot$ Carbon flurooxide $\cdot$ Nanofluids $\cdot$ Thermal conductivity $\cdot$ Photoacoustics

\section{Introduction}

Nanofluids, initially defined as stable colloidal solutions of nanoparticles with sizes smaller than $100 \mathrm{~nm}$, is one of the most remarkable kind of two-phase media with exciting thermophysical properties [1, 2]. Water, oils, organic solvents and glycols are usually used as hosting liquids for the nanofluid preparation. Studying of thermal properties of the colloidal solutions is important for efficient development of their various multidisciplinary applications [3-7].

Experiments based on transient hot-wire [8-10], hot disk [11], $3 \omega$ method [12], thermal constants analyzer [13, $14]$, steady-state parallel plate $[7,15]$, temperature oscillations [16], cylindrical cells and thermal comparator [17] were carried out for thermal conductivity estimation of nanofluids. A lot of different models based on: specific heat transfer mechanisms $[7,18]$, influence of Brownian motion of nanoparticles [19, 20], nanoparticle aggregation and cluster formation [21, 22], impact of specific surface of the suspended nanoparticles [23], nanoparticle size [24], effects of interfacial thermal resistance [25-27] were developed for description of thermal conductivity enhancement of the nanofluids. Development of multiscale models and a better understanding of the effects and processes occurring at the nanoparticle/fluid interface are important to predict transport properties of the nanofluids for their numerous applications.

A lot of thermal transport studies in carbon-based nanofluids based on nanodiamonds, nanotubes and graphene were already carried out [28]. Due to relatively high thermal conductivity of the carbon nanomaterials [29, 30], their dispersion in liquids allowed to improve heat transfer efficiency of the nanofluids.

K. Dubyk, kateryna.dubyk@gmail.com | 1 Taras Shevchenko National University of Kyiv, 64/13, Volodymyrska Street, Kiev 01601 , Ukraine. ${ }^{2}$ Science Park Kyiv Taras Shevchenko University, 60, Volodymyrska Street, Kiev 01033, Ukraine. ${ }^{3}$ LEMTA, Université de Lorraine, CNRS, 54500 Nancy, France. ${ }^{4}$ Nanotechnology Institute of Lyon (INL), University of Lyon, CNRS, 69361 Lyon, France. ${ }^{5}$ Light Matter Institute, UMR-5306, Claude Bernard University of Lyon, CNRS, Université de Lyon, 69622 Villeurbanne cedex, France. 
Previously, synthesis of a new kind of luminescent carbon-based nanomaterial, named carbon flurooxide (CFO), by an electrochemical etching of silicon carbide in HF-based solutions, was reported [31]. The CFO is an organic-like species of $\mathrm{C}_{\mathrm{x}} \mathrm{H}_{\mathrm{y}} \mathrm{O}_{\mathrm{z}} \mathrm{F}_{\mathrm{t}}$ chemical composition, easily soluble in polar solvents (water, alcohols) and consisting of 1-10 nm jellyfish-shape particles, which could be considered either as organic macromolecules or as fewlayer graphene-like mesoparticles, surrounded by continuous organic chains on the edges. The structure of the CFO is akin to the structure of such irregular biopolymers, as humic acids, melanins, etc., that is why high biocompatibility of the CFO could be expected. Indeed, the CFO possess an amazing ability to penetrate easily inside the living cells, and these mesoparticls were already successfully used as fluorescent cell labels and sonosensitizers for theranostic application [32]. As for thermal transport in the CFO-based nanofluids, it has never been studied before. However, precise estimation of thermal conductivity of the nanofluids could be especially important for photothermal theranostic approaches involving the CFO mesoparticles.

In this paper, results of thermal conductivity measurements performed on the CFO-based nanofluids are reported. Polyethylene glycol, oil and water were selected as basic hosting liquids. Photoacoustic technique with piezoelectric detection was used for the first time as an efficient non-destructive and noncontact tool to study thermal transport in the nanofluids. In particular, dependence of thermal conductivity on concentration of the CFO mesoparticles was studied in details.

\section{Experimental details}

\subsection{Colloidal solution preparation}

The CFO mesoparticles were fabricated via electrochemical etching of low-resistivity grade $(0.7 \mathrm{~m} \Omega \mathrm{cm}) \mathrm{n}$-type $3 \mathrm{C}$-SiC bulk polycrystalline substrate, according to previously reported protocols [31,33]. In brief, the anodization was performed in HF (48\%)-ethanol (1:1, v/v) mixture for $3 \mathrm{~h}$ at $25 \mathrm{~mA} / \mathrm{cm}^{2}$. After the etching, the wafer was gently washed several times with deionized water and then naturally dried in ambient conditions. The brown layer of porous $\mathrm{SiC}$ and CFO mixture, formed onto the substrate after anodization, was collected by mechanical scratching giving a powder. To separate the $\mathrm{CFO}$ and porous $\mathrm{SiC}$, the powder was dispersed in ethanol, the porous $\mathrm{SiC}$ precipitate was removed by centrifugation, while the supernatant was allowed to dry in ambient conditions giving the CFO powder. Structural features as well as chemical composition of the fabricated CFO nanopowder are described in details elsewhere [31, 33]. Spatial model of the CFO mesoparticles, derived from the model structural formula [31] after molecular mechanics optimization (MM2 algorithm, Chem3D) is presented on Fig. 1. A planar graphene/fluorinated graphane core surrounded by bulky alkyl backbones bearing the carboxylates and other substituents can be seen. In order to fabricate a nanofluid sample, the CFO mesoparticles were dispersed in water, PEG-200 and Vaseline oil without using any surfactant and finally the formed solutions were ultrasonicated.

\subsection{Experimental measurements}

Thermal conductivities of the CFO-based nanofluids were evaluated from photoacoustic measurements using a sandwich structure shown in Fig. 2. The structure

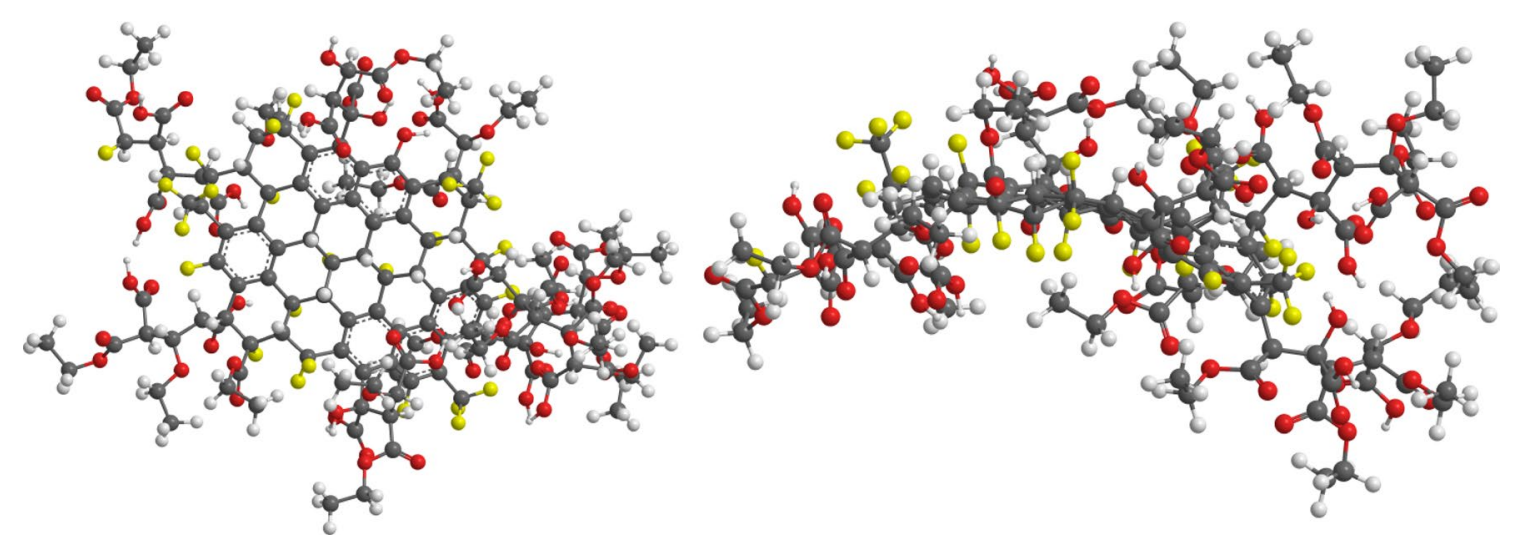

Fig. 1 Structural chemical model of a CFO mesoparticle. Color of atoms: C-grey, F-yellow, O-red, H-white 


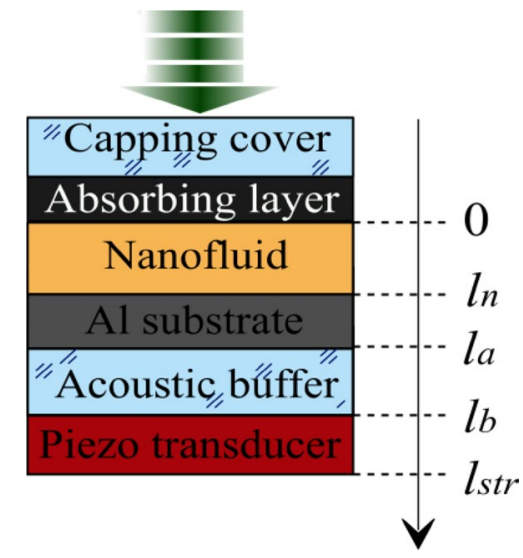

Fig. 2 Scheme of the multilayered structure for thermal conductivity measurements

containing the nanofluids is irradiated through a transparent capping cover by a laser of $405 \mathrm{~nm}$ wavelength with modulated intensity. The laser light is locally absorbed by a special absorbing layer. The laser-induced heating occurring in the layer is transferred through the studied fluid toward an aluminum substrate. The thermo-elastic stresses generated in the aluminum plate are transmitted through an acoustic buffer toward the piezoelectric transducer. Thus, the main mechanism of the photoacoustic signal formation is based on photothermal transformation and thermoelastic effect. Time delay between the heating beginning in the absorbing layer and the recorded bending of the whole system depends on thermal conductivity of the studied fluid. The characteristic coordinates of the sandwich components represented in Fig. 2 are the following: $I_{n}=140 \mu \mathrm{m}, I_{a}=1040 \mu \mathrm{m}, I_{b}=2040 \mu \mathrm{m}, I_{\text {str }}=2740$ $\mu \mathrm{m}$. Thicknesses of the nanofluid, aluminum substrate, acoustic buffer and piezoelectric transducer can be easily deduced from the corresponding values of the coordinates mentioned above. Photoacoustic signals were detected in a frequency range from 2 to $23 \mathrm{~Hz}$. Amplitude-frequency characteristic (AFC) and phase-frequency characteristic (PFC) are measured with a lock-in amplifier. Typically, AFC characterizes portion of energy which transfer through liquid layer, and PFC defines time delay between starting of heating and arising of a registered response depending on the modulation frequency.

\subsection{Experimental data treatment}

Theoretical simulation of AFC and PFC of the generated photoacoustic signals allows estimation of thermal properties of the studied nanofluids. Quasi-stationary approximation of the signal formation in the sandwich structure is considered. Firstly, spatial distribution of the variable component of the temperature $\theta$ in the structure is calculated.
The temperature profiles can be evaluated by solving heat diffusivity equation [34] transformed according to thermal wave formalism:

$\frac{d}{d z}\left(k(z) \frac{d \theta}{z}\right)-i \omega c \rho \theta=0$,

where $\omega$ is the angular frequency, $c, \rho, k$ are the specific heat, the density and the thermal conductivity in the considered structure, respectively. The assumption that almost all power of irradiation is absorbed in the thin absorbed layer was used:

$\left.k \frac{\partial \theta}{\partial z}\right|_{z=0+0}=\left.k \frac{\partial \theta}{\partial z}\right|_{z=0-0}+l$,

here $l$ is the intensity of the absorbed radiation.

Additionally, limitation of temperature at the infinity was also assumed:

$\left.\theta\right|_{z \rightarrow \pm \infty}=0$

Equation (1) was numerically solved for the multilayered structure, considering the conditions (2) and (3) with the use of finite difference method.

Then, spatial distribution of the thermoelastic stresses arising in the sandwich structure are described in frames of a rigid normal assumption, as reported earlier [35]. The voltage appearing on electrodes of the piezoelectric transducer can be expressed as function of the elastic stresses detected by the transducer [35]:

$U(\omega) \sim \int_{I_{b}}^{I_{s t r}} \sigma(z) d z=\int_{I_{b}}^{I_{s t r}} \frac{E(z)}{1-v(z)} \int_{I_{s t r}}^{I_{n}} \frac{\alpha_{T} E \theta(s, \varpi)}{1-v(s)} G(z, s) d s d z$,

where $\sigma(z)$ is the spatial distribution of the thermoelastic stresses, $E$ is the Young's modulus, $v$ is the Poisson's coefficient, $\alpha_{T}(s)$ is the thermal expansion coefficient of the material. This equation can be used to fit experimental amplitude-frequency and phase-frequency dependencies of the photoacoustic responses.

\section{Results and discussions}

Typical amplitude-frequency and phase-frequency characteristics of the photoacoustic signals for water, PEG-200 and Vaseline oil are shown in Fig. 3. One can see variation of the curve slopes which can be related to different physical properties of the studied liquids ensuring different conditions for propagation of the thermal perturbation through the sandwich structure. Such features of the curve behavior allow reliable estimation of the liquid/nanofluid thermal conductivity. Experimental amplitude and phase 


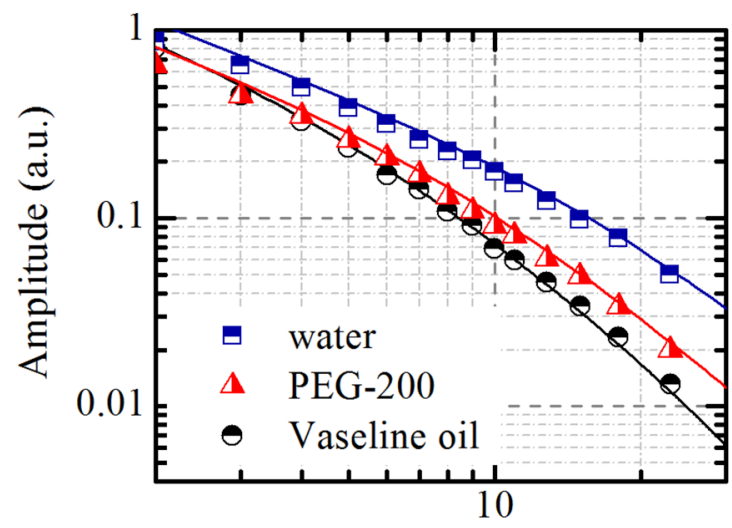

Frequency $(\mathrm{Hz})$

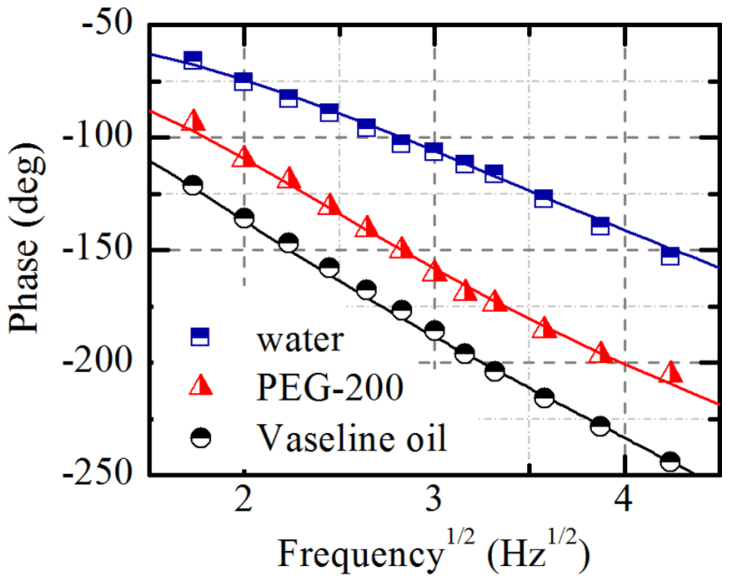

Fig. 3 Typical amplitude-frequency and phase-frequency characteristics of the photoacoustic signals for basic liquids. The scatters correspond to the experimental points and the solid lines are the theoretical fittings

frequency characteristics were fitted with the experimental ones with use of least square method. Consequently, thermal conductivity of nanofluid was evaluated as a fitting parameter [36]. Thermal conductivity values for water, PEG-200 and Vaseline oil were found to be, respectively: $0.57 \pm 0.04,0.19 \pm 0.02$ and $0.14 \pm 0.02 \mathrm{~W} / \mathrm{m} \mathrm{K}$. These values are in good agreement with those reported in literature: $0.6 \mathrm{~W} / \mathrm{m} \mathrm{K}$ for water [37], $0.2 \mathrm{~W} / \mathrm{m} \mathrm{K}$ for PEG-200 [38] and $0.13 \mathrm{~W} / \mathrm{m} \mathrm{K}$ for Vaseline oil [39].

The amplitude-frequency dependencies of the CFObased nanofluids using PEG-200 as basic liquid with various particles concentrations expressed in terms of volume fraction are shown in Fig. 4a). The global curve behavior is similar to that shown in Fig. 3 for the initial basic liquids. In addition, the higher the volume fraction of the CFO is, the higher the amplitude of the recorded photoacoustic signal is. Thermal conductivities of the CFO nanofluids deduced from the fitting of the amplitude-frequency dependencies (in water, PEG and Vaseline oil) as function of volume fraction of the mesoparticles can be seen in Fig. 4b). Significant thermal conductivity rise depending on the CFO concentration can be observed. The maximum thermal conductivity enhancement is observed at the 0.16 volume fraction in Vaseline oil (up to $85 \%$ ). It should be noted that the thermal (a)

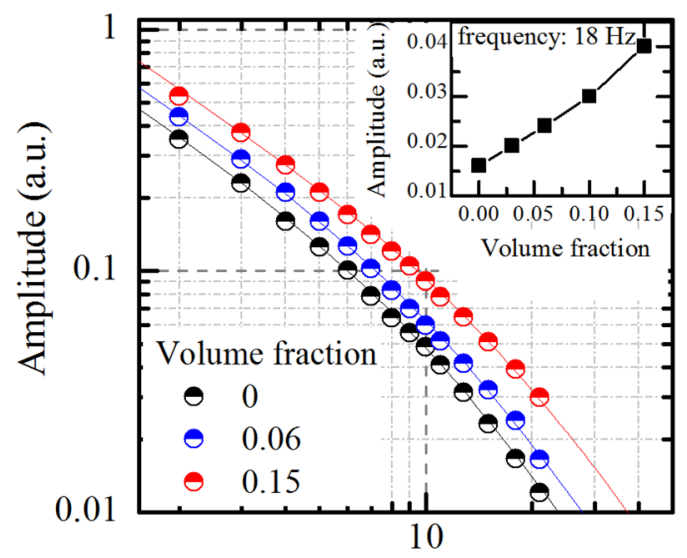

Frequency $(\mathrm{Hz})$ (b)

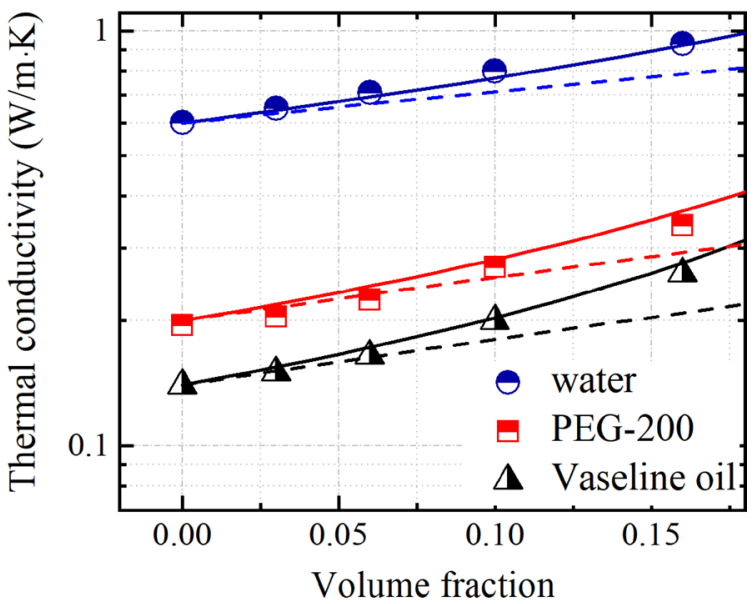

of volume fraction of the mesoparticles. The scatters correspond to the values deduced from the photoacoustic characteristics, the dashed and the solid lines are the theoretical fittings according to the classical Maxwell model and modified Maxwell model, respectively 
conductivity of the nanofluids nonlinearly increases with increasing of volume fraction.

For description of such thermal conductivity increase, the effective medium approximation based on Maxwell model was considered. The classical Maxwell model is used for solid-liquid mixtures with relatively large size of particles or their low concentrations [23]. Maxwell's formula shows that the effective thermal conductivity of nanofluids depends on (i) thermal conductivity of spherical particle $\left(k_{p}\right)$, (ii) thermal conductivity of base fluid $\left(k_{f f}\right)$ and (iii) volume fraction of the solid particles $(\varphi)$ :

$k_{\text {eff }}=k_{f f} \frac{k_{p}+2 k_{f l}+2\left(k_{p}-k_{f l}\right) \varphi}{k_{p}+2 k_{f l}-\left(k_{p}-k_{f f}\right) \varphi}$

The effective thermal conductivity of nanofluids evaluated from the classical Maxwell model (Eq. (5)) should linearly increase along the volume fraction rise in a low concentration range. This fact is, indeed, in a good agreement with our obtained experimental results for the volume fraction range $<0.06$ (see dashed lines in Fig. 4b). In contrast, when the volume fraction is $>0.06$, a significant deviation of the classical Maxwell model from the experimental results with highly pronounced non-linear rise of thermal conductivity can be stated.

Thus, a modified Maxwell model was applied for a better description of the non-linear thermal conductivity behavior. Indeed, according to a three component model of $\mathrm{Yu}$ and Choi [40], a nanofluid can be represented as a composition of nanoparticles, fluid and an interfacial layer surrounding the nanoparticles. This layer appears as a result of interfacial interaction between atoms of solid and fluid [41, 42]. Being dependent on nanoparticle volume fraction, continuously increasing specific area of the interfacial layers is supposed to be responsible for the overall enhancing the thermal conductivity $[18,22,43,44]$. Thus, the addition of the interfacial layer leads to a modified Maxwell model with corresponding replacement of the thermal conductivity of a solid particle $k_{p}$ by a new thermal conductivity of a particle $k_{p, \text { eff }}$ including its adjacent interfacial layer:

$k_{p, \text { eff }}=k_{p} \frac{\left(2(1-\beta)+(1+\gamma)^{3}(1+2 \beta)\right) \beta}{-(1-\beta)+(1+\gamma)^{3}(1+2 \beta)}$

here $\beta=k_{f} / k_{p}$ is the ratio of the interfacial layer thermal conductivity $\left(k_{f l}\right)$ to particle thermal conductivity $\left(k_{p}\right)$, $\gamma=h / r$ is the ratio of the layer thickness $(h)$ and the nanoparticle radius $(r)$.

Thus, the resulting effective thermal conductivity of a nanofluid can be expressed as follows:

$k_{\text {eff }}=k_{f f} \frac{k_{p, \text { eff }}+2 k_{f l}+2(1+\gamma)^{3}\left(k_{p, e f f}-k_{f f}\right) \varphi}{k_{p, \text { eff }}+2 k_{f l}-(1+\gamma)^{3}\left(k_{p, \text { eff }}-k_{f f}\right) \varphi}$ where $k_{f l}$ is the thermal conductivity of the basic hosting fluid, $k_{p, \text { eff }}$ is the effective thermal conductivity of the nanoparticles (according to Eq. 6) and $\varphi$ is the volume fraction of nanoparticles in the nanofluids.

An excellent agreement between the modified Maxwell model according to the Eq. 7 (see continuous lines) and experimental data from Fig. 4b) allows to conclude on a key role of the interface nanolayer [45] in thermal transport of the CFO-based nanofluids.

\section{Conclusion}

In summary, thermal conductivity of the oil-, PEG- and water-based nanofluids composed by CFO mesoparticles were evaluated by means of photoacoustic approach with piezoelectric detection for the first time. The obtained experimental values of thermal conductivities are situated in the range from 0.1 to $1 \mathrm{~W} / \mathrm{m} \mathrm{K}$. Significant non-linear thermal conductivity enhancement (up to $85 \%$, in comparison with initial basic liquids) depending on volume fraction of the CFO mesoparticles were described in frames of modified Maxwell model. In particular, such thermal conductivity behavior was explained by influence of nanolayers at the interface between the mesoparticles and hosting liquid.

Acknowledgements This research work was carried out in frames of CARTHER Project (Proposal \#690945) of Marie Skłodowska-Curie Research and Innovation Staff Exchange program (Horizon 2020 Framework Program). The publication contains the results obtained in the frames of the research work "Features of photothermal and photoacoustic processes in low-dimensional silicon-based semiconductor systems" (Ministry of Education and Science of Ukraine, State Registration Number 0118U000242). This work has been partially funded by the CNRS Energy unit (PEPS Cellule ENERGIE 2019) through the project ImHESurNaASA. MI wants also to acknowledge the partial financial support of the scientific pole EMPP of University of Lorraine. The authors are grateful to Dr. Svitlana Gryn from Taras Shevchenko National University of Kyiv for the helpful discussion.

Funding This study was funded by Marie Skłodowska-Curie Research and Innovation Staff Exchange program (Proposal 690945), by Ministry of Education and Science of Ukraine (State Registration Number 0118U000242), by CNRS Energy unit (PEPS Cellule ENERGIE 2019) through the project ImHESurNaASA, and by Scientific pole EMPP of University of Lorraine.

\section{Compliance with ethical standards}

Conflict of interest The authors declare that they have no conflict of interest.

\section{References}

1. Eastman JA, Choi SUS, Li S, Yu W, Thompson LJ (2001) Anomalously increased effective thermal conductivities of ethylene glycol-based nanofluids containing copper nanoparticles. Appl Phys Lett 78:718-720. https://doi.org/10.1063/1.1341218 
2. Sheikholeslami M, Hayat T, Alsaedi A (2016) MHD free convection of $\mathrm{Al}_{2} \mathrm{O}_{3}$-water nanofluid considering thermal radiation: a numerical study. Int J Heat Mass Transf 96:513-524. https://doi. org/10.1016/j.ijheatmasstransfer.2016.01.059

3. Ryabchikov YV, Alekseev S, Lysenko V, Bremond G, Bluet J (2013) Photoluminescence thermometry with alkyl-terminated silicon nanoparticles dispersed in low-polar liquids. Phys Status Solidi Rapid Res Lett 7:414-417. https://doi.org/10.1002/pssr.20130 7093

4. Ryabchikov YV, Alekseev SA, Lysenko V, Bremond G (2013) Photoluminescence of silicon nanoparticles chemically modified by alkyl groups and dispersed in low-polar liquids. J Nanoparticle Res 15:1535. https://doi.org/10.1007/s11051-013-1535-3

5. Taylor R, Coulombe S, Otanicar T, Phelan P, Gunawan A, Lv W, Rosengarten G, Prasher R, Tyagi H (2013) Small particles, big impacts: a review of the diverse applications of nanofluids. J Appl Phys 113:011301. https://doi.org/10.1063/1.4754271

6. Pryazhnikov MI, Minakov AV, Rudyak VY, Guzei DV (2017) Thermal conductivity measurements of nanofluids. Int J Heat Mass Transf 104:1275-1282. https://doi.org/10.1016/j.ijheatmasstrans fer.2016.09.080

7. Cardellini A, Fasano M, BozorgBigdeli M, Chiavazzo E, Asinari $\mathrm{P}$ (2016) Thermal transport phenomena in nanoparticle suspensions. J Phys: Condens Matter 28:483003. https://doi. org/10.1088/0953-8984/28/48/483003

8. Assael MJ, Metaxa IN, Kakosimos K, Constantinou D (2006) Thermal conductivity of nanofluids: experimental and theoretical. Int J Thermophys 27:999-1017. https://doi.org/10.1007/s1076 5-006-0078-6

9. Penaas JRV, Ortiz De Zarate JM, Khayet M (2008) Measurement of the thermal conductivity of nanofluids by the multicurrent hot-wire method. J Appl Phys 104:1-8. https://doi. org/10.1063/1.2970086

10. Roder HM (1934) A transient hot wire thermal conductivity apparatus for fluids. J Res Natl Bur Stand 86(1981):457. https:// doi.org/10.6028/jres.086.020

11. Warzoha RJ, Fleischer AS (2014) Determining the thermal conductivity of liquids using the transient hot disk method. Part I: establishing transient thermal-fluid constraints. Int J Heat Mass Transf 71:779-789. https://doi.org/10.1016/j.ijheatmasstrans fer.2013.10.064

12. Dong-Wook Oh JSL, Jain A, Eaton JK, Goodson KE (2008) Thermal conductivity measurement and sedimentation detection of aluminum oxide nanofluids by using the $3 \omega$ method. Int J Heat Fluid Flow 29:1456-1461. https://doi.org/10.1016/j.ijhea tfluidflow.2008.04.007

13. Jiang W, Ding G, Peng H (2009) Measurement and model on thermal conductivities of carbon nanotube nanorefrigerants. Int J Therm Sci 48:1108-1115. https://doi.org/10.1016/j.ijthermals ci.2008.11.012

14. Zhu D, Li X, Wang N, Wang X, Gao J, Li H (2017) Dispersion behavior and thermal conductivity characteristics of $\mathrm{Al}_{2} \mathrm{O}_{3}-\mathrm{H} 2 \mathrm{O}$ nanofluids. Dispersion behavior and thermal conductivity characteristics. Curr Appl Phys. https://doi.org/10.1016/j.cap.2007.12.008

15. Trisaksri V, Wongwises $S$ (2007) Critical review of heat transfer characteristics of nanofluids. Renew Sustain Energy Rev 11:512523. https://doi.org/10.1016/j.rser.2005.01.010

16. Das SK, Putra N, Thiesen P, Roetzel W (2003) Temperature dependence of thermal conductivity enhancement for nanofluids. J Heat Transf 125:567-574. https://doi.org/10.1115/1.15710 80

17. Paul G, Chopkar M, Manna I, Das PK (2010) Techniques for measuring the thermal conductivity of nanofluids: a review. Renew Sustain Energy Rev 14:1913-1924. https://doi.org/10.1016/j. rser.2010.03.017
18. Wang $X$, Mujumdar AS (2015) Critical review of heat transfer characteristics nanofluids. Heat Transf Charact Nanofluids Rev. https://doi.org/10.1016/j.rser.2005.01.010

19. Bhattacharya P, Saha SK, Yadav A, Phelan PE, Prasher RS (2004) Brownian dynamics simulation to determine the effective thermal conductivity of nanofluids. J Appl Phys 95:6492-6494. https ://doi.org/10.1063/1.1736319

20. Koo J, Kleinstreuer C (2004) A new thermal conductivity model for nanofluids. J. Nanoparticle Res 6:577-588. https://doi. org/10.1007/s11051-004-3170-5

21. Duan F, Kwek D, Crivoi A (2011) Viscosity affected by nanoparticle aggregation in $\mathrm{Al}_{2} \mathrm{O}_{3}$-water nanofluids. Nanoscale Res Lett 6:248. https://doi.org/10.1186/1556-276X-6-248

22. Keblinski JAEP, Phillpot SR, Choi SUS (2002) Mechanisms of heat flow in suspensions of nano-sized particles (nano fluids). Int J Heat Mass Transf 45:855-863. https://doi.org/10.1016/S0017 -9310(01)00175-2

23. Vadasz $P$ (2005) Heat conduction in nanofluid suspensions. J Heat Transf 128:465-477. https://doi.org/10.1115/1.2175149

24. Chopkar M, Sudarshan S, Das PK, Manna I (2008) Effect of particle size on thermal conductivity of nanofluid. Metall Mater Trans A 39:1535-1542. https://doi.org/10.1007/s11661-007-9444-7

25. Li J, Kleinstreuer C (2008) Thermal performance of nanofluid flow in microchannels. Int J Heat Fluid Flow 29:1221-1232. https ://doi.org/10.1016/j.ijheatfluidflow.2008.01.005

26. Ge Z, Cahill DG, Braun PV (2006) Thermal conductance of hydrophilic and hydrophobic interfaces. Phys Rev Lett. https://doi. org/10.1103/physrevlett.96.186101

27. Hu L, Desai T, Keblinski P (2011) Determination of interfacial thermal resistance at the nanoscale. Phys Rev B 83:195423. https ://doi.org/10.1103/PhysRevB.83.195423

28. Younes H, AlGhaferi A, Saadat I, Hong H (2016) Nanofluids based on carbon nanostructures. In: Silva A (ed) Advanced carbon nanostructures. InTech, Rijeka. https://doi.org/10.5772/64553

29. Balandin AA, Ghosh S, Bao W, Calizo I, Teweldebrhan D, Miao F, Lau CN (2008) Superior thermal conductivity of single-layer graphene. Nano Lett 8:902-907. https://doi.org/10.1021/nl073 1872

30. Balandin AA (2011) Thermal properties of graphene, carbon nanotubes and nanostructured carbon materials. Nat Mater 10:569-581. https://doi.org/10.1038/nmat3064

31. Alekseev S, Korytko D, lazykov M, Khainakov S, Lysenko V (2015) Electrochemical synthesis of carbon fluorooxide nanoparticles from 3C-SiC substrates. J Phys Chem C 119:20503-20514. https ://doi.org/10.1021/acs.jpcc.5b06524

32. Kharin A, Syshchyk O, Geloen A, Alekseev S, Rogov A, Lysenko V, Timoshenko V (2015) Carbon fluoroxide nanoparticles as fluorescent labels and sonosensitizers for theranostic applications. Sci Technol Adv Mater 16:44601

33. Gryn S, Nychyporuk T, Bezverkhyy I, Korytko D, lablokov V, Lysenko V, Alekseev S (2018) Mesoporous SiC with potential catalytic application by electrochemical dissolution of polycrystalline 3C-SiC. ACS Appl Nano Mater 1:2609-2620. https://doi. org/10.1021/acsanm.8b00301

34. Carslaw HS, Jaeger JC (1959) Conduction of heat in solids, 2nd edn. Clarendor Press, Oxford

35. Isaiev M, Andrusenko D, Tytarenko A, Kuzmich A, Lysenko V, Burbelo $R$ (2014) Photoacoustic signal formation in heterogeneous multilayer systems with piezoelectric detection. Int J Thermophys 35:2341-2351. https://doi.org/10.1007/s10765-014-1652-y

36. Dubyk K, Chepela L, Lishchuk P, Belarouci A, Lacroix D, Isaiev $M$ (2019) Features of photothermal transformation in porous silicon based multilayered structures. Appl Phys Lett 115:021902

37. Ramires $M$, Nieto $C$, Nagasaka $Y$, Nagashima A, Assael M, Wakeham W (1995) Standard reference data for the 
thermal conductivity of water. J Phys Chem Ref Data. https:// doi.org/10.1063/1.555963

38. Sun Q, Yuan Y, Zhang H, Cao X, Sun L (2017) Thermal properties of polyethylene glycol/carbon microsphere composite as a novel phase change material. J Therm Anal Calorim 130:17411749. https://doi.org/10.1007/s10973-017-6535-6

39. Kothandaraman CP, Subramanyan S (2013) Heat and mass transfer data book, 8th edn. New Academic Science, New Delhi

40. Yu W, Choi SUS (2003) The role of interfacial layers in the enhanced thermal conductivity of nanofluids: a renovated Maxwell model. J Nanoparticle Res 5:167-171

41. Isaiev M, Burian S, Bulavin L, Gradeck M, Termentzidis K (2016) Efficient tuning of potential parameters for liquid-solid interactions. Mol Simul 42:910-915. https://doi.org/10.1080/08927 022.2015 .1105372

42. Isaiev M, Burian S, Bulavin L, Keblinski P, Termentzidis K (2018) Gibbs adsorption impact on a nanodroplet shape: modification of Young-Laplace equation. J Phys Chem B 122:3176-3183
43. Xue Q, Xu W-M (2005) A model of thermal conductivity of nanofluids with interfacial shells. Mater Chem Phys 90:298-301. https ://doi.org/10.1016/j.matchemphys.2004.05.029

44. Xie H, Fujii M, Zhang X (2005) Effect of interfacial nanolayer on the effective thermal conductivity of nanoparticle-fluid mixture. Int J Heat Mass Transf 48:2926-2932. https://doi.org/10.1016/j. ijheatmasstransfer.2004.10.040

45. Loulijat $H$, Zerradi $H$ (2019) The effect of the liquid layer around the spherical and cylindrical nanoparticles in enhancing thermal conductivity of nanofluids. J Heat Transf. https://doi. org/10.1115/1.4042329

Publisher's Note Springer Nature remains neutral with regard to jurisdictional claims in published maps and institutional affiliations. 